\title{
Medical Image Denoising using Fast Discrete Curvelet Transform
}

\author{
P Anandan ${ }^{1}$, A Giridhar ${ }^{2}$, E Iswarya Lakshmi ${ }^{3}$, P Nishitha $^{4}$ \\ ${ }^{1}$ Vel Tech Rangarajan Dr. Sagunthala R\&D Institute of Science and Technology, Chennai, Tamilnadu, India, \\ anand.phd.dip@gmail.com \\ ${ }^{2}$ Vel Tech Rangarajan Dr. Sagunthala R\&D Institute of Science and Technology, Chennai, Tamilnadu, India, \\ ${ }^{3}$ Vel Tech Rangarajan Dr. Sagunthala R\&D Institute of Science and Technology, Chennai, Tamilnadu, India, \\ ${ }^{4}$ Vel Tech Rangarajan Dr. Sagunthala R\&D Institute of Science and Technology, Chennai, Tamilnadu, India,
}

\begin{abstract}
Now- a- days, medical field plays a very crucial role in our daily life, as a part of it MRI (Magnetic resonance imaging) scans, CT (computed tomography) images, Ultrasound images etc. of the victim which are one of the main things that are to be determined correctly based on which the patient's condition is concluded and treated. The main problem here occurs is for the original image where the image gets noisy and the features of the original image are lost due to many factors. So, here in our paper, we instigate the method of image denoising technique which helps to eliminate the noisy observations and other disturbances and reconstructs the original image very accurately. The image denoising is one of the important preprocessing steps in medical field image processing analysis. For this denoising method, we are going to use the Fast Discrete Curvelet Transform which is a multi-scale geometric transform and is designed to signify the image or video sequences at different scales and angles. also the performances of it by using fast Fourier discrete curve-let transform which is based on ridge-let analysis theory for denoising procedures and makes recommendations with the help of adaptive threshold algorithm which is applied on the image and gets the original image with effectiveness also retrieves the important detail features in the image and also the quality of the image to be recovered by using the parameters like Peak Signal to Noise Ratio (PSNR), Mean Square Error (MSE).
\end{abstract}

Key words: Curvelet transform, Adaptive Thresholding, PSNR, MSE, Ridgelet analysis, denoising, MRI, CT, Ultrasound image.

\section{INTRODUCTION}

In image processing techniques, one of the fundamental challenges is image denoising in which the main aim is to get the original Image by suppressing all the noise from the noise-contaminated version of the image.
This image denoising technique is mainly used and focuses on the area of medical applications and it plays an important role in an extensive range of applications such as image registration, image classification, and image segmentation, in which we obtain the unique image content, is decisive for improved performance or operation[15]. This noise reduction problem for images is to eliminate the noise from an image to recover a clean and clear image in this the image acts as the input which is the sum of relevant pixels of the unclean image(noisy image) corresponding to the pixels of the clean image(noise-free).For example, applied denoising methods to deblurring, inpainting, and demosaicing similarly many cases can be obtained from with strength of noise and distribution of it over the image.

The less computational complexity algorithm proposed by Qiang Guo uses nonlocal self-similarity and low-rank approximation (LRA) which includes a grouping of similar image patches to be low-rank. Then factorization of each group is done by using Singular Value Decomposition(SVD) [1][9].Back projection is performed to cross-verify grouping errors if any and ultimately de-noised image is formed by aggregating the factorized groups.LRA in SVD makes the algorithm less computational complex by avoiding representation of image patches. Due to the optimal energy compaction property of SVD,this algorithm provides quantitative quality results in peak signal to noise ratio and feature-similarity index parameters. Taking the advantage of nonlocal redundancy and LRA by using a block-matching technique to construct matrices of the low-rank group for weakening the noise makes the algorithm simple and efficient technique for image de-noising. (An efficient SVD based method for image denoising).

Medical image denoising using convolutional denoising Auto encoders.In which the medical image denoising, though deep learning techniques are limited to sample size and computations [2][12].Gondara done an experiment with small sample size constructed using convolutional layers for competent medical image denoising. The denoising performance is increased by boosting the sample size by 
combining heterogeneous images. This algorithm can reconstruct the image by increasing the corruption levels so high such that differentiation of noise and signal is not possible to the human eye [14].

Another existing method for processing images in the photon-limited regime proposed by Yoann Altmann, Reuben Aspden, Miles Padgett, and Steve McLaughlin, in which they used Bayesian denoising method and by measuring the pixel measurement which summed up to follow Bernoulli distribution [3][5]. This method provides smoothness of image and increases potentiality and positivity of the intensity. A gamma Markovrandom field was proposed to design an intensity prior model abled and captured the spatial and temporary structures (A Bayesian Approach to Denoising of Single-Photon Binary Images)

In a patch-based denoising method DCNN for seismic image proposed by Yushu Zhang, Hongbo Lin, Vue li. Though the method of $(\mathrm{CNN})$ convolution neural network for image denoising it is empowered with a specific noise level that cannot deal with images, they introduced PDCNN (patchbased denoising convolution neural network) which clusters the overlapping patches of noisy images [4][23][11]. The capability of PDCNN is demonstrated on synthetic and field seismic images.

\section{FAST DISCRETE CURVELET TRANSFORM}

Curve-let transform is a multi-scale geometric transform that can signify edges and curves originalities more competently than traditional wavelets as wavelets have the disadvantage of poor directionality [8][10][22][23]. Although the complex wavelet transform is proof for directional selectivity, it is difficult to design complex wavelets with good filter characteristics and perfect reconstruction properties[16][18].

At first, the first-generation curve-let transform was introduced as a ridge-let transform. Later second-generation curve-let transform was introduced but faced two drawbacks that are it is highly redundant and it is not optimal for the sparse approximation of curve features beyond c 2 singularities [13][17]. Later fast and less redundant curve-let transforms were introduced. The newly implemented curve-let transform known as Fast Discrete curve-let transform (FDCT) is implemented in two ways using wrapping function and unequally spaced Fast Fourier transforms (USFFT). In USFFT, curvelet coefficients are obtained by an irregular sampling of Fourier samples whereas in the wrapping method coefficients are obtained by successive translations through wrap-around technique.

The curvelet transform function is represented as

$$
\mathrm{c}(\mathrm{j}, 1, \mathrm{k})=<f, \varphi_{\mathrm{j}, 1, \mathrm{k}}>(1)
$$

Where $\varphi_{\mathrm{j}, 1, \mathrm{k}^{-}}$is the curvelet; $\mathrm{j}$ - is scale; 1 - is direction; $\mathrm{k}$ - is position

\subsection{Fast discrete curvelet transform through wrapping technique}

In the Curvelet transform with wrapping technique, the oversampled coefficients are obtained by

$$
\mathrm{C}^{\mathrm{D}, \mathrm{O}}(\mathrm{j}, 1, \mathrm{k})=\frac{1}{\mathrm{n}^{2}} \sum_{\mathrm{n}_{1}, \mathrm{n}_{2} \in \mathrm{R}_{\mathrm{j}, 1}} \mathrm{f}\left[\mathrm{n}_{1}, \mathrm{n}_{2}\right] \tilde{\mathrm{u}}_{\mathrm{j}, 1}\left[\mathrm{n}_{1}, \mathrm{n}_{2}\right] \mathrm{e}^{2 \pi \mathrm{i}\left(\frac{\mathrm{k}_{1} \mathrm{n}_{1}}{\mathrm{R}_{1, j}}+\frac{\mathrm{k}_{2} \mathrm{n}_{2}}{\mathrm{R}_{2, \mathrm{j}}}\right)}(2)
$$

Here D, $\mathrm{O}$ are Digital and Oversampled

$R_{\mathrm{j}, 1}$ is a rectangle of size $R_{1, j}{ }^{*} R_{2, j}$. The coefficients $C^{D, O}(j, l, k)$ is obtained by discrete convolution of a curvelet and signal $f\left(t_{1}, t_{2}\right)$. The rectangle's dimensions $R_{1, j}$, and $R_{2, j}$ are generally large. In the wrapping method, $R_{1, j}$ and $R_{2, j}$ are replaced by the dimensions $\left(\mathrm{L}_{1, \mathrm{j}}\right.$ and $\left.\mathrm{L}_{2, \mathrm{j}}\right)$ of the parallelogram Pjl. This type is just as like that of relabeling of the frequency samples represented as

$$
\begin{aligned}
& \mathrm{n}_{1}^{\prime}=\mathrm{n}_{1}+\mathrm{m}_{1} \mathrm{~L}_{1, \mathrm{j}} \\
& \mathrm{n}_{2}^{\prime}=\mathrm{n}_{2}+\mathrm{m}_{2} \mathrm{~L}_{2, \mathrm{j}}
\end{aligned}
$$

The 2- dimensional inverse FFT of the wrapped array takes the form

$$
\mathrm{c}^{\mathrm{D}}(\mathrm{j}, 1, \mathrm{k})=\frac{1}{\mathrm{n}^{2}} \sum_{\mathrm{n}_{1}=0}^{\mathrm{Ll},{ }^{-1}} \sum_{\mathrm{n}_{1=0}}^{\mathrm{Ll}, \mathrm{j}^{-1}} \mathrm{~W}\left(\tilde{\mathrm{U}}_{\mathrm{j}, 1}, \hat{\mathrm{f}}\right)\left[\mathrm{n}_{1}, \mathrm{n}_{2}\right] \mathrm{e}^{2 \pi \mathrm{i}\left(\mathrm{k}_{1} \mathrm{n}_{1} / \mathrm{L}_{1, j}+\mathrm{k}_{2} \mathrm{n}_{2} / \mathrm{L}_{2, j}\right)}(5)
$$

At first, the curvelet transform is performed to find curvelet coefficients as follows.

\subsection{Steps concerned in wrapping based curvelet transform}

1. Firstly, the Fast Fourier transform is applied to the input image.

2. Secondly, the Curvelets are obtained at a given scale 's' and orientation ' $n$ '.

3. Then the Fast Fourier Transform (FFT) is divided into small sets.

4. Each set is interpreted to the origin for all set.

5. Then the small set that is in parallelogram shape is wrapped to rectangular shape positioned at the origin.

6. Finally, inverse FFT is performed and Curvelet coefficients are composed as an array.

After finding curvelet coefficients, inverse curvelet transform is performed to get the denoised image

\subsection{Steps concerned in inverse curvelet transform based on wrapping}

1. For every curvelet coefficient array, the inverse FFT is found.

2. The inverse process unties the rectangular support to the first form orientation.

3. The translation is then carried out to obtain the original position.

4. The entire curvelet array is then stored.

5. Then alltogether the translated and the stored curvelet arrays are added.

6. The reconstructed image is finally obtained by taking inverse FFT. 


\section{THRESHOLDING TECHNIQUES}

According to digital image processing, thresholding is known for segmenting the images .it is used to create a binary image that consists of black and white pixels that have two intensity values. The threshold is a fixed value that draws a boundary line between two sets of data [20][21].

The thresholding is one of the important techniques of image segmentation methods in the field of image processing which is used to isolate the objects by converting to grey-scale images [24].

The thresholding is calculated by using an iterative method. It is a non-linear operation. The threshold value is estimated by the clustering method of the histogram.

1. Firstly, separate the pixels into two clusters as per threshold

2. Find the mean of clusters

3. Square the difference between the means and

4. Multiply the by the number of pixels in one cluster times the number in the other.

This depends only on the difference between the means of the 2 clusters, thus avoiding having to calculate differences between individual intensities and therefore the cluster means. The optimal threshold is that the one that maximizes the between-class variance (or, conversely, minimizes the within-class variance).

\section{1 types of thresholding techniques}

The types of threshold techniques in the field of image processing are

1.Global thresholding

2. Local thresholding

3.Adaptive thresholding

\section{Global thresholding}

It is a technique in which it consists of setting an intensity value such that the values of all pixels having intensity are below the threshold value of one phase. It is good at the degree of intensity separation between the two peaks in the image

\section{Local thresholding}

It is a technique that is used to convert the image which consists of greyscale to black and white pixel images. In this technique, every pixel of the image is analyzed concerning neighboring pixels

\section{Adaptive thresholding}

It is also known as dynamic thresholding [6]. It is used to separate desirable foreground image objects from the background based on the difference in pixel intensities of each region Our paper is concerned with adaptive threshold methods apart from all the other thresholding techniques
[7][19]. It is one of the best techniques in the field of digital image processing. mat lab is also used along with the adaptive threshold algorithm for calculation part and result(output) of the image which is based on Fast Discrete Curvelet Transform. The conventional thresholding techniques use a global threshold for all pixels, whereas adaptive thresholding changes the threshold value all over the image. Comparatively, the adaptive threshold chooses the accurate method for binarizing an image based on the contrast, texture, resolution, etc. of an image. Thus, the adaptive threshold method is adapted in this paper for the image denoising of MRI, CT, Ultrasound scans in the medical field application

\section{PROPOSED METHODOLOGY}

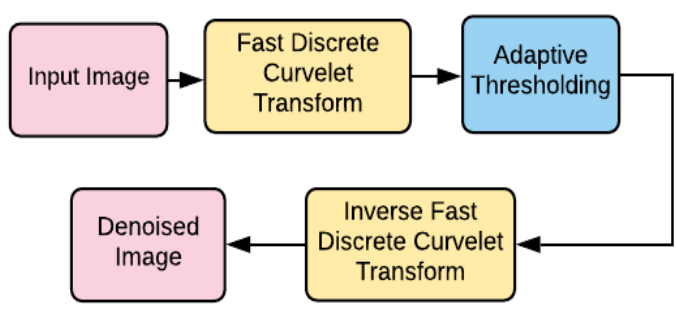

Figure 1: Block diagram of proposed image denoising scheme

The objective of this work is to denoise the input image and to achieve high PSNR and minimum MSE without affecting the quality of the image. In the proposed method, we are using a Fast discrete curvelet transform through wrapping technique and adaptive thresholding for image denoising. Curvelet transform can represent curve singularities and edges more efficiently than any other transforms as it is the multi-scale geometric transform.

The basic steps involved in the proposed method are shown in figure 1. At first, the input image is decomposed using a fast discrete curvelet transform. The decomposition is carried out and curvelet coefficients are obtained using the wrapping technique. Then, suitable threshold is applied using adaptive thresholding, to neglect the coefficients below the threshold value, and to preserve the coefficients above the threshold value.The curvelet coefficient is calculated based on the threshold. Inverse curvelet transform is then applied using the inverse wrapping technique. The denoised image is finally obtained.

\section{PERFORMANCE PARAMETERS}

In our paper, we used the PSNR and MSE parameters for determining the quality of the regained image after denoising and also the other qualities like noise removal, quality of reconstructed image, etc.

Peak Signal to Noise Ratio is meant to be the original image and the error is compared as the noisy observation

$$
\text { PSNR }=\frac{20 * \log _{10}(\text { Max.Pixel value })}{\sqrt{\mathrm{MSE}}}
$$


Mean Square Error is a parameter which calculates the cumulative squared error between the original image and the compressed image

$$
M S E=\frac{1}{N} \sum_{i} \sum_{j}(f(i, j)-\llbracket F(i, j)) \rrbracket^{2}(7)
$$

Where $\mathrm{N}$ denotes the total number of pixels.

$\mathrm{f}(\mathrm{i}, \mathrm{j})$ denotes the pixel value in the original image.

$F(i, j)$ denotes the pixel value in the reconstructed image.

\section{RESULTS AND DISCUSSION}

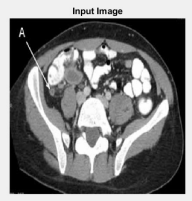

a

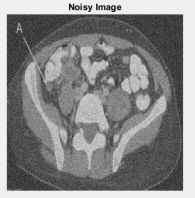

b

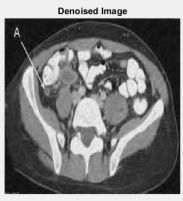

c
Figure 2: CT Abdomen image (a) Input(b) Noisy(c) Denoised

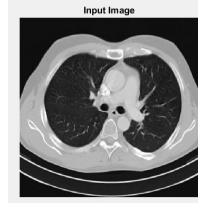

a

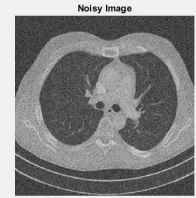

b

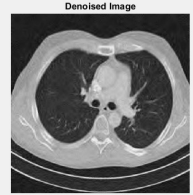

Figure 3: CT Chest image (a) Input(b) Noisy(c) Denoised

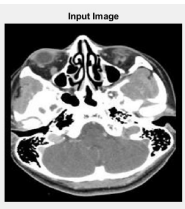

a

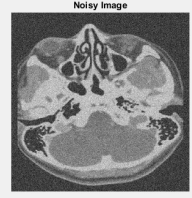

b

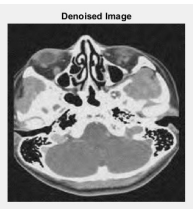

c
Figure 4: CT Brain image (a) Input(b) Noisy(c) Denoised

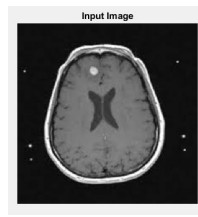

a

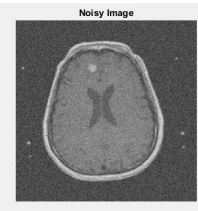

b

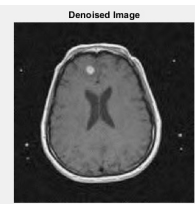

c
Figure 5: MRI Brain image (a) Input(b) Noisy(c) Denoised

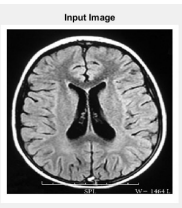

a

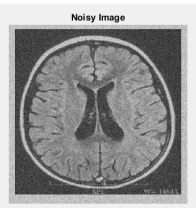

b

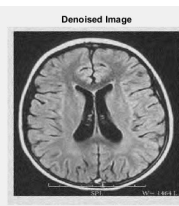

$\mathrm{c}$
Figure 6: MRI Cerebrum image (a) Input(b) Noisy(c) Denoised

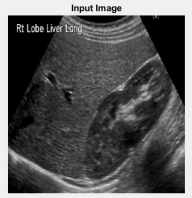

a

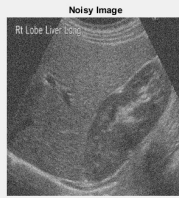

b

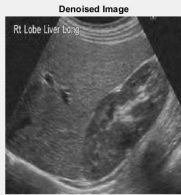

c
Figure 7: Ultrasound Liver image (a) Input(b) Noisy(c) Denoised

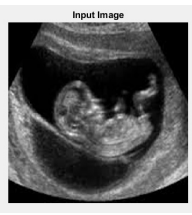

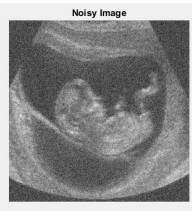

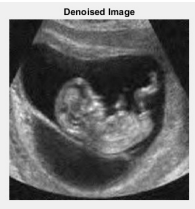

Figure 8: Ultrasound fetus image (a) Input(b) Noisy(c) Denoised

Table 1: Comparison of PSNR(dB) values

\begin{tabular}{|c|c|c|c|}
\hline Input Image & Proposed & DWT & DCT \\
\hline CT Abdomen image & 30.34 & 27.3 & 21.3 \\
\hline CT Chest image & 30.43 & 26.8 & 19.4 \\
\hline CT Brain image & 29.7 & 23.4 & 16.7 \\
\hline MRI Brain image & 33.2 & 28.6 & 18.6 \\
\hline MRI Cerebrum image & 28.2 & 22.7 & 16.8 \\
\hline Ultrasound Liver image & 30.17 & 24.3 & 19.3 \\
\hline Ultrasound fetus image & 32.6 & 26.7 & 16.1 \\
\hline
\end{tabular}

Table 2: Comparison of MSE values

\begin{tabular}{|c|c|c|c|}
\hline Input Image & Proposed & DWT & DCT \\
\hline CT Abdomen image & 0.6 & 0.81 & 0.9 \\
\hline CT Chest image & 0.58 & 0.72 & 0.91 \\
\hline CT Brain image & 0.68 & 0.8 & 0.92 \\
\hline MRI Brain image & 0.3 & 0.63 & 0.8 \\
\hline MRI Cerebrum image & 0.98 & 0.73 & 0.89 \\
\hline Ultrasound Liver image & 0.62 & 0.77 & 0.87 \\
\hline Ultrasound fetus image & 0.35 & 0.66 & 0.82 \\
\hline
\end{tabular}




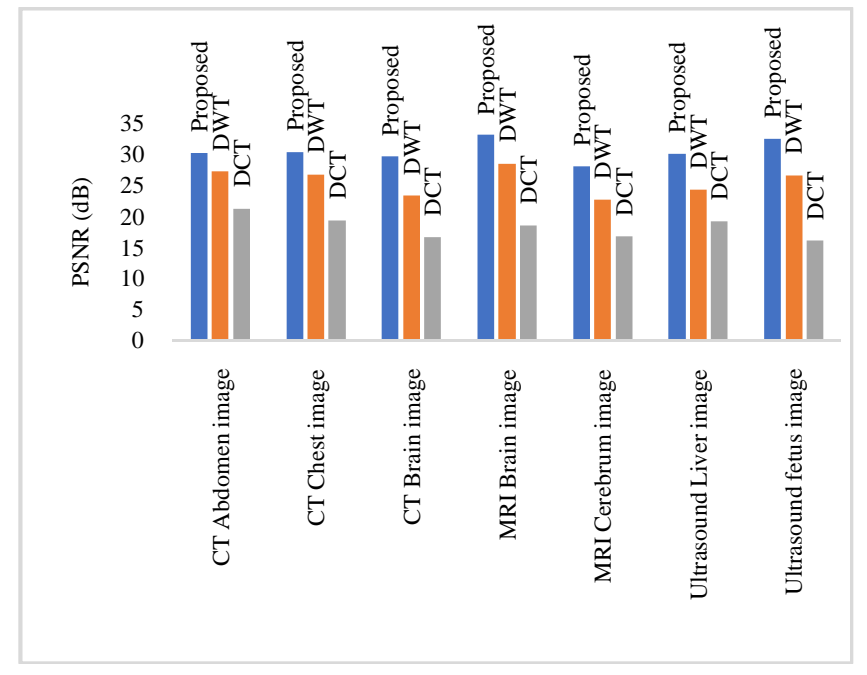

Figure 9: Comparison of PSNR Values

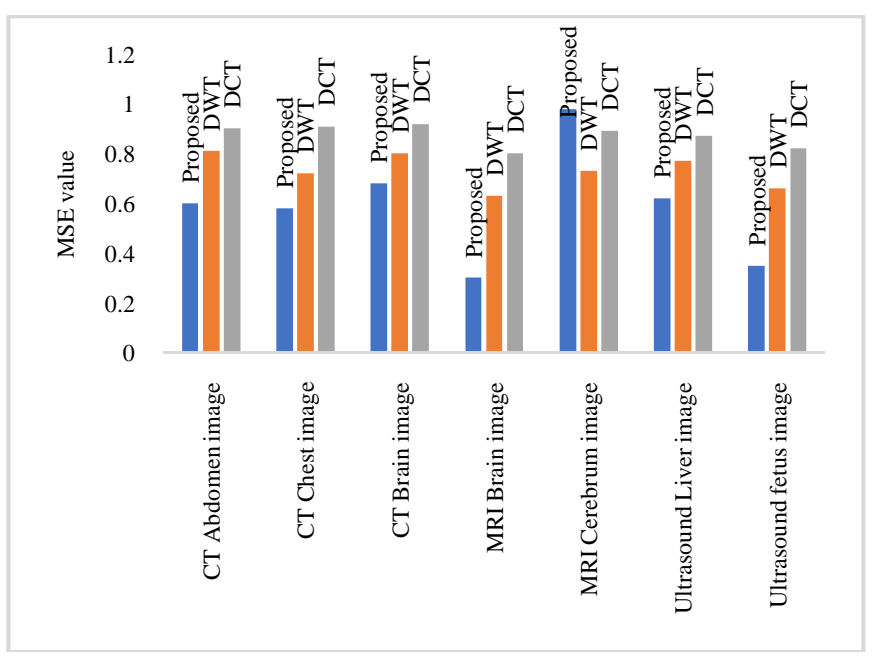

Figure 10: Comparison of MSE values

The performance of the proposed algorithm is tested on different medical images of the database available at www.kaggle.com. Simulation output obtained for different input images are shown in Figure 2 to Figure 8.Figure 2 shows the output for the CT Abdomen image. Figure 3 shows the output for the CT Chest image. Figure 4 shows the output for the CT Brain image. Figure 5 shows the output for the MRI Brain image. Figure 6 shows the output for MRI Cerebrum image. Figure 7 shows the output for the Ultrasound Liver image. Figure 8 shows the output for the Ultrasound fetus image. A comparison of performance parameter PSNR is shown in Table 1 and the comparison of performance parameter MSE is shown in Table 2. Table 1 shows that the proposed method gives the higher PSNR compare to DWT and DCT. Table 2 shows that the proposed method gives lesser MSE compare to DWT and DCT. Figure 9 shows the comparison of PSNR values of different input images. Figure 10 shows the comparison of MSE values of different input images.

\section{CONCLUSION}

The proposed method in this paper of image denoising uses an adaptive threshold algorithm with the help of a fast discrete curvelet transform which gives a reconstructed image without compromising the quality of the original image and removes the noisy observations from the image. This technique can be successfully implemented in denoising images like MRI, Ultrasound, CT images as a part of medical field image processing Also the curvelet transform is one of the efficient methods which gives the better result as compared to DWT and DCT. Whereas, the PSNR and MSE are the two parameters used for measurement of quality and error involved in image and also compare the original with the denoised image (output image). The Proposed algorithm may be extended for denoising images of different fields by keeping the appropriate threshold for various noises.

\section{REFERENCES}

1. Qiang Guo, Caiming Zhang, Yunfeng Zhang, and Hui Liu, An efficient SVD based method for image denoising, IEEE transactions on circuits and systems for video technology, Vol. 26, No. 5, pp. 868-880, 2016. https://doi.org/10.1109/TCSVT.2015.2416631

2. L. Gondara, Medical Image Denoising Using Convolutional Denoising Autoencoders,2016 IEEE 16th International Conference on Data Mining Workshops (ICDMW), Barcelona, pp. 241-246, 2016.

3. Yoann Altmann, Member, Reuben Aspden, Miles Padgett, and Steve McLaughlin, A Bayesian Approach to Denoising of Single-Photon Binary Images,IEEE transactions on computational imaging, vol.3,no. 3.,pp. 460-471,2017.

https://doi.org/10.1109/TCI.2017.2703900

4. Yushu Zhang, Hongbo Lin, Yue Li and Haitao Ma, A Patch Based Denoising Method Using Deep Convolutional Neural Network for Seismic Image, IEEE Access, vol.7, pp. 883-894, 2019.

5. $P$ Anandan, A Manikandan, $R$ S Sabeenian, D Bharathidhasan, Non subsampled contourlet transform based video compression using Huffman and run length encoding for multimedia applications, International Journal of Advanced Trends in Computer Science and Engineering, Volume 9, No. 3, 2020. https://doi.org/10.30534/ijatcse/2020/93932020

6. N B Rais, M S Hanif, Rmtiaz A. Taj, Adaptive Thresholding Technique for Document Image Analysis, IEEE 8th International Multitopic Conference Proceedings, pp. 61 - 66, 2004.

7. H Wang, L Qian, J Zhao, An image denoising method based on fast discrete curvelet transform and total variation, IEEE 10th International Conference on Signal Processing Proceedings, pp. 1040-1043, 2010. 
8. G I. Salama, A M. Elmolla, A novel Video Compression using fast curvelet transform,International Journal of computer Science and Telecommunications., Vol. 6, no. 3, 2015.

9. P Anandan, S Kokila, S, Karuppaiah, Video Compression using APRS and Fast Discrete Curvelet Transform for Mobile Devices, SYLWAN Journal, Volume 164, No. 6, pp. 2-16, 2020.

10.J.D. Pujari,Dhasrath.K.Bhadangkar, Identification and Recognition of Facial Expressions Using Image Processing Techniques, International Journal of Emerging Trends in Engineering Research, Volume 5, No.5, pp. 1 - 10, May 2017.

11. P Anandan, RS Sabeenian,Medical Image Compression using Fast Discrete Curvelet Transform and Arithmetic coding, Circuits and Systems, Vol. 7, pp. 2059-2069, 2016. https://doi.org/10.4236/cs.2016.78179

12. M. Gooroochurna, DKerrb, K Bouazza-Maroufb, Image Processing of Planar Digital Curves Using a Chain Code-based Technique for Edge Characterization, International Journal of Emerging Trends in Engineering Research, Vol. 4, No. 7, pp. 50 - 63, July 2016.

13. Dr. L.M. Varalakshmi1, Radha Ramalingam, License Plate Character Recognition using Advanced Image Processing Techniques and Genetic Algorithm, International Journal of Emerging Trends in Engineering Research, Volume 3, No.4, pp. 8 - 13, April 2015.

14. P Anandan, R S Sabeenian, Fabric defect detection using Discrete Curvelet Transform, Elsevier Procedia Computer Science, Volume 133, pp. 1056-1065, 2018. https://doi.org/10.1016/j.procs.2018.07.058

15. Jesusimo L. Dioses Jr., Discrimination of Civet Coffee Using Image Processing and Machine Learning, International Journal of Emerging Trends in Engineering Research, Volume 8. No. 4, pp. 1067 - 1072 April 2020. https://doi.org/10.30534/ijeter/2020/19842020

16. Xiang, Qian \& Pang, Xuliang, Improved Denoising Auto-Encoders for Image Denoising, IEEE 11th International Congress on Image and Signal Processing, Biomedical Engineering and Informatics, 2018.

17. Donoho D L, Duncan M R, Digital curvelet transform: strategy, implementation and experiment, Proc. SPIE 4056, Wavelet Applications VII, vol.6, pp .12-30,2000.

18. $P$ Anandan, RS Sabeenian, Image Compression Techniques using Curvelet, Contourlet, Ridgelet and Wavelet Transforms, A Review, Biometrics and Bioinformatics, Volume 5, Issue 5, pp. 267-270, 2013.

19. S. Nasrin, M. Z. Alom, R. Burada, Medical Image Denoising with Recurrent Residual U-Net (R2U-Net) base Auto-Encoder, IEEE National Aerospace and Electronics Conference, pp. 345-350, 2019.
20. Xin Wang, Moving window based doblehaar wavelet transform for image processing, IEEE transactions on image processing, vol.15, issue. 9, pp. 302-317,2006.

21. G.Gilboa, N.Sochen, Y.Y.Zeevi, Image enhancement and denoising by complex diffusion process, IEEE transactions on pattern analysis and machine intelligence, Vol.26, Issue.8, pp. 171-192, 2004. https://doi.org/10.1109/TPAMI.2004.47

22. T. Jiang, X. Zhao, W. Ding, J. Chen, Improved image denoising method based on Curvelet transform, IEEE International Conference on Information and Automation, Harbin, pp. 1086-1090, 2010.

23. S. Zhen-gang and L. Qin-zi, Pulmonary CT image denoising algorithm based on curvelet transform criterion, IEEE 7th International Symposium on Microwave, Antenna, Propagation, and EMC Technologies, pp. 520-524, 2017. https://doi.org/10.1109/MAPE.2017.8250909

24. P Anandan, G Tejeswi, S Vijayalakshmi, J Srilakshmi, Detection of Goblet Cells to diagnose Barrett's Esophagus using Contourlet Transform and GLSM approach, 2020 International Conference on Computer Communication and Informatics (ICCCI), pp. 1-5, 2020. 